\title{
Downregulation of PLK4 Expression Induces Cellular Dormancy in Colorectal Cancer via Mesenchymal-to-epithelial Transition
}

\section{Xiangdong Tian}

Tianjin Tumor Hospital

\section{Dongming Liu}

Tianjin Tumor Hospital

\section{Dejun Zhou}

Tianjin Tumor Hospital

Lisha Qi

Tianjin Tumor Hospital

\section{Zhiqiang Han}

Tianjin Tumor Hospital

\section{Ziye Chen}

Tianjin Tumor Hospital

Wenchen Gong

Tianjin Tumor Hospital

\section{Qiuping Dong}

Tianjin Tumor Hospital

\section{Yi Luo}

Tianjin Tumor Hospital

Liwei Chen

Tianjin Tumor Hospital

\section{Jiefu Wang}

Tianjin Tumor Hospital

\section{Hua Li}

Tianjin Tumor Hospital

\section{Peng Liu}

Tianjin Tumor Hospital

\section{Lu Chen}

Tianjin Tumor Hospital

\section{Qiang Zhao}

Tianjin Tumor Hospital

Hua Guo ( $\sim$ guohua@tjmuch.com )

Tianjin Medical University Cancer Institute and Hospital

\section{Research}

Keywords: PLK4, colorectal cancer, MET, dormancy, prognosis

Posted Date: August 17th, 2020 
DOI: https://doi.org/10.21203/rs.3.rs-59372/v1

License: (c) (i) This work is licensed under a Creative Commons Attribution 4.0 International License. Read Full License 


\section{Abstract}

Background: Reactivation of dormant tumor cells is a critical step in the recurrence of many cancers, including colorectal cancer (CRC). Polo-like kinases 4 ( $P L K 4)$, a central regulator of the cell cycle and proliferation, is a validated oncogene in tumorigenesis. However, the roles of PLK4 in tumor cell dormancy and reactivation still need to be further explored.

Methods: The expression level of PLK4 was determined by immunohistochemical staining, Western blotting (WB) and quantitative real-time PCR (qRT-PCR). PLK4-dependent clinicopathological risk factors and the prognosis of CRC were characterized with 122 clinical samples. The roles of PLK4 in tumor cell dormancy, cell cycle progression, proliferation and invasion were determined by molecular and cell biology methods in vitro and in vivo.

Results: The expression of PLK4 was dramatically increased in CRCs and positively correlated with aggressive tumor behavior and clinicopathological risk factors. Downregulation of PLK4 expression contributed to restoring phenotypically aggressive tumor cells to a quiescent state, and this transformation was likely regulated by mesenchymal-to-epithelial transformation (MET) progression in vitro and in vivo.

Conclusions: This study elucidates the mechanisms involving PLK4 depletion in the induction and maintenance of CRC dormancy, which are very important in terms of both clinical significance and application value.

\section{Introduction}

CRC is one of the most commonly diagnosed cancers among men and women worldwide[1]. The US 2019 cancer statistics report shows that both the morbidity and mortality of CRC rank in the top five among those of all cancer types[2]. Local recurrence and distant metastasis are the main risk factors affecting the prognosis of patients with CRC, especially those with stage IV CRC. Remarkably, up to $65 \%$ of patients with stage IV CRC have relapsed disease after treatment with curative intent, while on $30 \%$ of patients with stage I-III CRC experience relapse after similar treatment[3]. Although modern curative treatments have already greatly improved the overall survival (OS) and disease-free survival (DFS) of patients with CRC in recent years, the success of these treatment strategies is generally based on early detection of primary and recurrent CRC[4, 5]. Therefore, further exploration of the molecular mechanism underlying CRC recurrence and metastasis and identification of candidate biomarkers of these processes are urgently needed and important for improving the CRC survival rate.

Recurrence and metastasis usually originate from residual and disseminated tumor cells[6]. However, nearly one-third of CRC cases already have disseminated disease lodged in target organs, such as the liver and lymph nodes, by the time of diagnosis[7]. Although the application of neoadjuvant or preventive chemotherapy greatly contributes to destroying disseminated tumor cells, $25-40 \%$ of patients with CRC still experience local or distant metastatic relapse, sometimes years or even decades after apparent cure[8]. Previous studies have shown that a subpopulation of disseminated tumor cells can escape radiotherapy, chemotherapy and immune monitoring or developing a "dormant" phenotype[9]. These dormant tumor cells can be long-term latent in the circulatory systems or target organs[10]. During this period, residual cancer cells are clinically undetectable, and patients usually have no clinical manifestations or symptoms[11]. Evidence has shown that the reactivation of dormant tumor cells is governed by complex interactions between disseminated initiating cells and the microenvironment of the target organ[12]. When dormant tumor cells are reactivated and gain the ability to reenter the cell cycle, cancer recurrence occurs[11, 13]. However, the mechanisms underlying the alternation between phases of dormancy and reactivation are still largely unknown.

Generally, dormant tumor cells are referred to as special-state tumor cells in which the cells exist in an equilibrium between proliferation and death[11]. The main characteristic of dormant tumor cells is G0/G1 cell cycle arrest, which is achieved by activation of quiescence programs[11, 14]. Accordingly, the proliferation marker MKI6711], ERK and p38 MAPK activities[15] and the cyclin-dependent kinase (CDK) inhibitor $P 2 X[14]$ are often used to label and identify dormant tumor cells. To date, proteins that regulate cell cycle reprogramming have been fairly well studied. Among these proteins, PLK4, a member of the 
Polo-like kinase family, was identified as an important regulator of many cell cycle-related events[16, 17]. Due to the critical role of PLK4 in controlling cell cycle progression, its importance in oncogenesis can be hypothesized[18, 19]. Our previous study showed that downregulated PLK4 expression in neuroblastoma (NB) cells mediates MET, and decreased PLK4 expression in NB is indicative of prolonged survival[20]. Consistently, Kazazian et al. reported that depletion of PLK4 suppressed breast cancer progression and induced an epithelial phenotype in poorly differentiated cancer cells[21]. Notably, recent evidence suggests a role for $P L K 4$ as a proliferation promoter in colorectal carcinogenesis mediated by activating the Wnt/ $\beta$-catenin signaling pathway[22]. However, controlling the cell cycle and regulating cell proliferation may not be the only functions of $P L K 4$ relevant to tumorigenicity. The potential association between $P L K 4$ and tumor cell phenotypic transformation needs to be further explored.

In this study, we verified a new molecular mechanism involving PLK4 in the induction of CRC progression. The results showed that the proliferation and metastasis of CRC cells were largely dependent on PLK4 expression. Knockdown of PLK4 expression in CRC cells triggered the MET process and impaired migration and invasion, which was accompanied by G0/G1 cell cycle arrest and low Ki67 expression, characteristics of dormancy. Furthermore, when we reversed the MET process caused by the deregulation of PLK4, dormant CRC cells were reactivated. Thus, these findings imply a dependence of PLK4driven alternation between dormancy and reactivation on MET and epithelial-to-mesenchymal transformation (EMT). PLK4 is a promising target to induce tumor cells into permanent dormancy for clinical CRC treatment.

\section{Materials And Methods}

\subsection{Patients and tissue specimens}

Tissue samples were tested and verified as CRC or adjacent normal tissue by two independent pathologists, and the CRC samples were staged based on the 8th edition of the American Joint Committee on Cancer (AJCC) Cancer Staging Manual. This study was approved by the Ethics Committee of the Tianjin Medical University Cancer Institute and Hospital (Approval No. bc2020073) and was consistent with the ethical guidelines of the Helsinki Declaration and approved by the Ethics Committee.

\subsection{Cell culture}

SW480 and HCT8 cells were purchased from the Type Culture Collection of the Chinese Academy of Sciences (Shanghai, China). Due to culture requirements $\left(37^{\circ} \mathrm{C}, 5 \% \mathrm{CO} 2\right)$, the two cell lines were cultured in complete DMEM (Corning, New York, USA) supplemented with a $1 \%$ penicillin-streptomycin solution (PS; HyClone, Logan, Utah, USA) and 10\% fetal bovine serum (FBS; PAN-Seratech, Edenbach, Germany).

\subsection{Cell transfection}

To acquire lentiviral particles, expression plasmids (sh-Ctrl and sh-PLK4) and packaging plasmids (VSVG and $\triangle \mathrm{R}$ ) were transfected into HEK293T cells supplemented with PEI (Polysciences, Warrington, USA). SW480 and HCT8 cells were used for transfection. Polybrene (Solarbio, Beijing, Chia) was applied as the transfection reagent. Stably transfected SW480 and HCT8 cell lines were obtained under puromycin (Gibco, New York, USA) selection.

\subsection{Immunohistochemical staining}

Immunohistochemistry (IHC) was applied to assess the protein level of PLK4 in paraffin-embedded samples of CRC tissue according to previously described methods[23]. An anti-PLK4 antibody was used at the concentration of 1:200 (Abcam, Cambridge, UK). The IHC score was used to examine the correlation between PLK4 expression and OS in CRC patients. In CRC patients, the IHC score was also used in correlation analysis for some clinicopathological factors. The percentage of PLK4-positive cells was evaluated from 0 to 3 ( 0 , no positive cells; $1,<30 \%$ positive cells; $2,30-60 \%$ positive cells; and 3 , $60 \%-100 \%$ positive cells). The PLK4 staining intensity was scored across a range of four grades ( 0 , no positive staining; 1 , 
weakly positive staining; 2 , moderately positive staining; and 3, strongly positive staining). Finally, the IHC staining intensity and percentage scores were integrated to calculate the IHC score.

\subsection{RNA extraction, cDNA synthesis, and qRT-PCR}

Total RNA was separated from adherent cells with TRIzol reagent (Ambion, Texas, USA). According to a qRT-PCR kit (Takara, Tokyo, Japan), cDNA was synthesized by reverse transcription of the isolated RNA. The amplification reaction was executed with designed primers based on the manufacturer's instructions (Takara). The primer sequences are shown in Table 1.

Table 1

The primer sequences in this manuscript

\begin{tabular}{|lll|}
\cline { 2 - 4 } & \multicolumn{3}{c}{ The primer sequences in this manuscript } \\
\hline PLK4 & GTACGATTCTGATAACCCC & G-primer \\
MKI67 & CTGACCCTGATGAGAGTGAGGG & TCTCCCCTTTTGAGAGGCGT \\
P15 & TGGGGTGGGAAAGTGGATTGCA & CCCAGTGCAGAGGTGTTCAGGTCT \\
P16 & GCTGCTCACCTCTGGTGCCAAA & ACCTGCGCACCATGTTCTCG \\
P27 & GGTTAGCGGAGCAATGCGCA & AACCGGCATTTGGGGAACCGTC \\
\hline LRG1 & GGGTCAGAACCAAGGGGTTT & GTCACAGGCCATTGATCCCA \\
\hline BMP7 & ACGAGGTGCACTCGAGCTT & GAAGCGTTCCCGGATGTAGT \\
\hline GAPDH & TGGTATCGTGGAAGGACTCA & CCAGTAGAGGCAGGGATGAT \\
\hline
\end{tabular}

\subsection{WB and antibodies}

The following antibodies were used: anti-PLK4 $(1: 1,000)$, anti-CCNE1 $(1: 1,000)$, anti-PCNA $(1: 1,000)$ and anti-vimentin $(1: 8,000)$ from Abcam, anti-N-cadherin (1:500) from Santa Cruz Biotechnology (Delaware, USA); anti-E-cadherin (1:500) from Sino Biological Inc. (Beijing, China); anti-CCND1 (1:1,000), anti-p-P38 (1:1,000), anti-P38 (1:1,000), anti-p-ERK $(1: 4,000)$ and anti-ERK $(1: 1,000)$ from Cell Signaling Technology (Danvers, USA); and anti-GAPDH from Bioss (Beijing, China). The steps for WB were based on previously described methods[23].

\subsection{CCK-8 assay}

According to the protocol of the CCK-8 assay, sh-PLK4- and sh-Ctr-transfected SW480 and HCT8 cells were plated in a 96well plate at a density of $1-4 \times 10^{3}$ cells per well. Six parallel wells were used for each independent group. Ten microliters of CCK-8 (Dojindo Laboratories, Kyushu, Japan) reagent was mixed in each well and incubated with the cells for $4 \mathrm{~h}$ at $37^{\circ} \mathrm{C}$ in $5 \%$ CO2. The optical density (OD) value at $450 \mathrm{~nm}$ was evaluated with an enzyme-labeling instrument. Cell viability was assessed on days $0,1,2$ and 3 .

\subsection{Colony formation assay}

For cell proliferation analysis, sh-PLK4- and sh-Ctrl-transfected CRC cells were seeded in 6-well plates (500-1,000 cells/well) and incubated at $37^{\circ} \mathrm{C}$ in $5 \% \mathrm{CO} 2$ for $1-2$ weeks. Once colonies were evident, the cells were stained with crystal violet and photographed with a digital camera.

\subsection{Apoptosis analysis}

CRC cells were washed with ice-cold PBS and then suspending in $200 \mu \mathrm{L}$ of $1 \times$ binding buffer, followed by staining for 15 minutes in the dark with Annexin V-APC (eBioscience, California, USA). Based on the cell number, 400-800 $\mu \mathrm{L}$ of $1 \times$ binding buffer was added and mixed, and then the cells were evaluated. The percentage of apoptotic cells was assessed with thecc (Millipore, Massachusetts, USA). 


\subsection{Cell cycle assay}

CRC cells were digested, mixed in $95 \%$ ethanol and incubated at $4{ }^{\circ} \mathrm{C}$ overnight. Following centrifugation and washing, the cells were incubated with $500 \mu \mathrm{L}$ of propidium iodide (PI; BD Biosciences, New Jersey, USA) and stained in the dark for 15 minutes. The cell samples were assessed on a FACS Aria flow cytometer (BD) with CellQuest software, and the results were evaluated with FlowJo software.

\subsection{Scratch assay}

CRC cells were harvested at a concentration of $1.5-2 \times 10^{6}$ cells per well in 6-well plates. The next day, an equal-width scratch was made through the CRC cell monolayer in the 6-well plates by using a $2.5-\mu \mathrm{L}$ pipette tip. Then, the cells were washed with PBS two or three times, and $2 \%$ FBS was mixed into the unsupplemented DMEM. The healing of the scratch was recorded by assessing the reductions in the distance between the edges measured at a suitable time $(3,6,9,12$, or $24 \mathrm{~h})$ and comparing those distances with the average distance measured at six stochastic locations at $0 \mathrm{~h}$. Images were captured at 0 and $24 \mathrm{~h}$ with a microscope at $10 \times$ magnification. Data are shown as the mean \pm SD.

\subsection{Migration and invasion assays}

For the migration and invasion assays, DMEM supplemented with $20 \%$ FBS was loaded into the lower chamber of a transwell system. A total of $1 \times 10^{5}$ cells in $2 \%$ FBS DMEM were plated on an 8 - $\mu$ m polyvinyl pyrrolidone-free polycarbonate filter membrane (Corning) for the migration assay. For the invasion assay, Matrigel-coated transwell chambers were incubated for more than $1 \mathrm{~h}$ at $37^{\circ} \mathrm{C}$. After incubating for approximately $24 \mathrm{~h}$ at $37^{\circ} \mathrm{C}$ in $5 \% \mathrm{CO} 2$, the migrated or invaded cells on the bottom were fixed with $4 \%$ paraformaldehyde (PFA) for 20 minutes and stained.

\subsection{Immunofluorescence}

In total, $3 \times 10^{4}$ cells were plated in each well of 12-well plates containing sterile coverslips. The following day, PBS was used to wash the coverslips. The cells were fixed with $4 \%$ PFA for 15 minutes, permeabilized in $0.25 \%$ Triton X-100 for 5 minutes and blocked in $3 \%$ BSA for $1 \mathrm{~h}$, all at ambient temperature. After incubation with primary antibodies at $4{ }^{\circ} \mathrm{C}$ overnight, the cells were stained with Alexa Fluor 488-conjugated or Alexa Fluor 546-conjugated secondary antibodies (Invitrogen, California, USA) separately at room temperature for $1 \mathrm{~h}$ in the dark. 4',6-Diamidino-2-phenylindole (Roche, Basel, Switzerland) staining of cell nuclei was then performed. Then, we captured images with a confocal laser scanning microscope (Olympus FV1000, Japan).

\subsection{In vivo experiments}

Four- to five-week-old nude mice (SPF Biotechnology Co., Ltd., Beijing, China) were acquired for xenograft animal assays (n $=8$ per group). sh-Ctrl/SW480 and sh-PLK4/SW480 cells were prepared, and $5 \times 10^{6}$ cells in $100 \mu \mathrm{L}$ of PBS were injected subcutaneously. Tumor volume was measured every three days using a Vernier caliper. To establish lung metastasis models, prepared sh-Ctrl/SW480 and sh-PLK4/SW480 cells (5 mice/group, $2 \times 10^{6} / \mathrm{mL} ; 100 \mu \mathrm{L}$ per mouse) were injected into the tail vein. After six weeks, the mice were sacrificed, and the lungs were harvested. The harvested lung tissues were fixed with formalin and embedded in paraffin. The tissues were used for hematoxylin and eosin (H\&E) staining.

\subsection{The Cancer Genome Atlas and Gene Expression Omnibus datasets}


Raw data from The Cancer Genome Atlas (TCGA; National Cancer Institute) and Gene Expression Omnibus (GEO; NCBI) databases for CRC were downloaded from the official database websites. Then, the original data were normalized with $R$ Studio. We evaluated the expression of PLK4 in tumor, adjacent normal tissue and polyp tissue samples from the TCGA, GSE41657 and GSE41258 mRNA expression datasets.

\subsection{Gene set enrichment analysis}

Gene set enrichment analysis (GSEA) was used to evaluate whether the PLK4 mRNA level was correlated with CRC biological features and signaling pathways, including tumor metastasis, proliferation, invasiveness, cell cycling, EMT and patient survival, on the basis of the GSE32323 dataset for CRC evaluated with GSEA 4.0.0 (The Broad Institute of MIT and Harvard).

\subsection{Statistical analyses}

Clinical data were processed and assessed by using SPSS 24.0 for Windows (SPSS Inc., Chicago, IL). The univariate KaplanMeier method and multivariate Cox method were used to evaluate the independent risk factors and survival curves of CRC patients. Spearman correlation analysis was used to examine the correlations between the PLK4 staining score and clinicopathological factors.

\section{Results}

\subsection{PLK4 is overexpressed in CRC tissues and associated with aggressive tumor behavior}

The expression level pattern of PLK4 between tumor and adjacent normal tissues was first analyzed with TCGA Pancancer datasets. Remarkably, we found that PLK4 expression was dramatically enhanced in the tumor tissues compared with the adjacent normal tissues in most cancer types, including CRC (Fig. 1A). Notably, the above results were further confirmed by comparing the expression of PLK4 in fifty paired CRC and adjacent normal tissues in TCGA datasets (Fig. 1B). Moreover, similar results were also found in the GEO CRC datasets (GSE41657 and GSE41258) (Fig. 1C and D). To further validate the expression of PLK4, we collected 16 CRC patient-matched fresh tumor and adjacent normal tissue pairs. Consistently, qRTPCR analysis of 5 paired CRC and adjacent normal tissue samples and WB analysis of another 11 paired CRC and adjacent normal tissue specimens indicated that 15 out of the 16 pairs of samples had higher levels of PLK4 expression in the tumor tissue (Fig. 1E and F). Collectively, these results suggested that PLK4 expression was upregulated in CRC tissues.

To characterize the role of PLK4 in CRC progression, we next performed GSEA based on the mRNA data of CRC samples in a GEO dataset (GSE32323). The results showed that high PLK4 expression was remarkably associated with early recurrence and a series of malignant behaviors related to an unfavorable prognosis for CRC (Fig. 1G-L). Briefly, high expression of PLK4 was observed in CRC samples ( $p=0.010$, Fig. 1G), and CRC samples with high expression of PLK4 also usually exhibited an activated cell cycle $(p=0.040$, Fig. $1 \mathrm{H})$ and proliferation $(p<0.0001$, Fig. $1 \mathrm{I})$ as well as enhanced invasiveness $(p=0.006$, Fig. $1 \mathrm{~J}$ ) and metastasis ( $p<0.0001$, Fig. $1 \mathrm{~K}$ ). In contrast, although the difference was not statistically significant, low expression of PLK4 was positively associated with a tendency toward a better prognosis, as demonstrated by prolonged recurrence-free survival ( $p=0.076$, Fig. 1L). Overall, increased expression of PLK4 was found to be a critical risk factor for the development of CRC, suggesting that PLK4 might play a vital role in CRC progression.

\subsection{PLK4 is essential in the cell cycle, proliferation, migration and invasion of CRC cells}

To understand how PLK4 affects CRC cells, we employed lentivirus-mediated siRNA expression to silence PLK4 expression in SW480 and HCT8 cells to further investigate the potential biological function of PLK4 in CRC progression. The efficiency 
of PLK4 knockdown was verified by WB and qRT-PCR (Fig. 2A and B). First, the cell cycle and apoptosis in SW480 and HCT8 cells were evaluated by flow cytometry (Fig. 2C-F). Dramatically, downregulation of PLK4 expression in SW480 and HCT cells directly resulted in G0/G1 cell cycle arrest (Fig. 2C and D). However, there was no remarkable difference in apoptosis or necrosis between the scrambled and PLK4-knockdown groups for these two cell lines (Fig. 2E and F). Consistently, CCK-8 and clone formation assays were applied to detect cell proliferation abilities and revealed that depletion of PLK4 considerably restricted the proliferation of CRC cells (Fig. $2 \mathrm{G}$ and $\mathrm{H}$ ). Surprisingly, we found that endogenous suppression of PLK4 expression further resulted in impaired cell migration and invasion not only in SW480 cells but also in HCT8 cells by using wound-healing (Fig. 2I), chemotaxis (Fig. 2J) and transwell assays (Fig. 2K).

Overall, we evaluated the effects on the cell cycle, proliferation, migration and invasion by generating PLK4-knockdown CRC cells, and the results indicated that downregulation of PLK4 expression reduced the malignancy of CRC cells in vitro. The CRC cell biological characteristics changed by PLK4 depletion strongly suggest an essential role for PLK4 in CRC progression.

\subsection{Knockdown of PLK4 expression promotes epithelial and dormant phenotypic transformation in CRC cells}

Next, we further explored the molecular mechanism underlying the PLK4-induced growth and invasiveness of CRC cells, which is closely related to CRC malignant progression. GSEA was applied to derive enrichment scores for CRC samples with high or low PLK4 expression in the GSE32323 dataset. Strikingly, positive enrichment of EMT was observed in the CRC samples with high PLK4 expression (Fig. 3A). Significant positive correlations between highly expressed PLK4 and enriched proliferation- and cell cycle-related genes, such as MKI67, PCNA and CCND1, were also observed in the same dataset (Fig. 3B). The above results suggested a regulatory role for PLK4 in promoting the EMT process and maintaining tumor cell proliferative activity in CRC.

Moreover, as shown in Fig. 3C, we noted a marked decrease in E-cadherin expression in SW480 and HCT8 cells with stable knockdown of PLK4 expression compared with the corresponding cells in the scrambled group. Additionally, the expression of $\mathrm{N}$-cadherin and vimentin, which are mesenchymal markers, was significantly increased. These data exhibited great consistency with the GSEA results and highly indicated that downregulation of PLK4 expression induced epithelial phenotypic transformation in CRC cells. Moreover, PLK4-depleted epithelial-phenotype CRC cells lost expression of PCNA, CCND1 and CCNE1, which are important indicators of cell proliferation and the cell cycle status (Fig. 3D). Notably, subsequent WB studies showed that the impaired proliferation and cell cycle arrest caused by PLK4 depletion were accompanied by a decreased p-ERK level and an increased p-P38 level (Fig. 3D). It is noteworthy that the p-P38 ${ }^{\text {high }} / \mathrm{p}$-ERK ${ }^{\text {low }}$ signaling ratio is already widely used in the identification of tumor cell dormancy, according to previous studies[24, 25]. Subsequently, signaling molecules favoring the acquisition of a dormant phenotype, such as $P 15, P 16, P 27, M K I 67, L R G 1$ and $B M P 7$, were further characterized by a qRT-PCR assay. Consistently, upregulated $P 15, P 16, P 27, L R G 1$ and $B M P 7 \mathrm{mRNA}$ expression in CRC cell lines was observed with PLK4 deficiency, whereas MKI67 mRNA expression exhibited the opposite trend[26]. Therefore, our data strongly supported the conclusion that knockdown of PLK4 expression induced MET and tumor cell quiescence in CRC cells. However, the relationship between MET and tumor cell dormancy, which is caused by PLK4 depletion in CRC cells, needs to be further clarified.

\subsection{MET reversal reactivates dormant PLK4 depleted CRC cells}

To further investigate the molecular mechanism linking PLK4 depletion with MET and tumor cell dormancy in CRC cells, we first employed TGF- $\beta$ to revert MET to EMT in PLK4-knockdown CRC cells. Previous studies have shown that TGF- $\beta$ is an effective inducer of the EMT process and is responsible for cancer metastasis in a variety of cancer types[27]. As expected, the expression of E-cadherin was suppressed by TGF- $\beta$ stimulation in PLK4-depleted SW480 and HCT8 cells compared with cells in the control group. However, at the same time, the expression of mesenchymal markers, such as $\mathrm{N}$-cadherin and vimentin, was increased (Fig. 4A). These results indicated that TGF- $\beta$ promoted mesenchymal phenotypic transformation in CRC cells with loss of PLK4 expression. Of note, we further found that the epithelial phenotypic reversal mediated by TGF- $\beta$ 
was accompanied by increases in the $\mathrm{p}-\mathrm{ERK} \mathrm{K}^{\text {high }} / \mathrm{p}-\mathrm{P} 38^{\text {low }}$ signaling ratio and PCNA, CCND1 and CCNE1 expression levels, which suggested that dormant CRC cells were reactivated within the MET to EMT process (Fig. 4A).

Next, we performed a series of functional experiments to verify the loss of tumor cell dormancy in PLK4-depleted CRC cells coincubated with TGF- $\beta$. We noted a striking reduction in the percentage of G0/G1-phase PLK4-depleted CRC cells upon stimulation with TGF- $\beta$, while the percentages of G2/M- and S-phase cells were enhanced (Fig. 4B and C). However, there was no significant effect on apoptosis in the groups (Fig. 4D and E). These data demonstrated that PLK4 silencing promoted G0/G1 cell cycle arrest, which was mitigated by TGF- $\beta$-induced EMT. Moreover, consistent with the previous results shown in Fig. 2, cell proliferation and migration were impaired in PLK4-downregulated CRC cells; however, these impairments could be partially rescued in the process of MET to EMT mediated by TGF- $\beta$ stimulation (Fig. 4F and G). All these results indicate a regulatory role for PLK4 in the transformation between tumor cell dormancy and reactivation, which is actually mediated by conversion between the MET and EMT processes.

\subsection{Silencing PLK4 in CRC cells facilitates dormancy and impairs metastasis in vivo}

Further in vivo experiments were performed to evaluate the influence of $P L K 4$ silencing on tumorigenesis and metastasis. PLK4-knockdown or vector-transfected cells were subcutaneously implanted into nu/nu mice. Remarkably, the results demonstrated that the SW480-shPLK4-derived tumors exhibited a slower growth rate than the control tumors (Fig. 5A). Furthermore, knockdown of PLK4 expression in SW480 cells significantly decreased tumor volumes and weights in the xenograft mouse model (Fig. 5B and C). These results suggest that loss of PLK4 expression is negatively correlated with the malignant progression of $\mathrm{CRC}$, which may be related to the dormancy of CRC cells.

Considering the regulatory role of PLK4 in the dormancy of CRC cells found in vitro, we further detected the cell cycle and apoptosis in xenograft tumors and compared the results between the shPLK4 and vector groups. Similar to the in vitro results, depletion of PLK4 in CRC cells induced G0/G1 cell cycle arrest without any changes in apoptosis (Fig. 5D and E). Notably, increases in the $\mathrm{p}-\mathrm{P} 38^{\text {high }} / \mathrm{p}$-ERK ${ }^{\text {low }}$ signaling ratio and E-cadherin expression were observed in tumors established with PLK4-knockdown cells, whereas the expression of vimentin, PCNA, CCND1 and CCNE1 was dramatically decreased (Fig. 5F). In addition, immunohistochemical staining for Ki67, E-cadherin, vimentin, p-ERK and p-P38 revealed results consistent with the WB data (Fig. 5G).

More interestingly, we created lung metastasis mouse models via tail vein injection of PLK4-depleted SW480 cells or vector cells. Metastatic tumor nodules were evaluated and quantified by a pathologist examining H\&E-stained sections.

Surprisingly, the incidence of metastatic lesions in the lungs was significantly lower in the shPLK4 group than in the control group (Fig. 5H). Together, these findings demonstrated that silencing PLK4 in CRC cells inhibited tumor growth and metastasis in xenograft models, revealing a critical role for PLK4 in the induction of MET and tumor dormancy.

\subsection{PLK4 inactivation identifies tumor dormancy and predicts a good prognosis in CRC}

To further characterize PLK4 in clinical CRC, another cohort of 122 patients with CRC was collected. The expression pattern of PLK4 was detected by IHC. Next, the Kaplan-Meier method and a Cox regression model were applied to explore the correlation between PLK4 and the prognosis of CRC patients. Multivariate analysis showed that increased expression of PLK4 and elevated serum carcinoembryonic antigen (CEA) levels ( $\geq 5 \mathrm{ng} / \mathrm{ml}$ ) were two independent risk factors for the prognosis of CRC (Fig. 6A and Table 2). Moreover, we divided these patients into two groups based on their IHC scores for PLK4. Notably, the 5-year OS rate of the patients with low PLK4 expression was significantly higher than that of the patients with high PLK4 expression, which demonstrated a crucial role for PLK4 in CRC progression (Fig. 6B). Surprisingly, we also found that the low PLK4 expression was highly consistent with p-P38 high/p-ERK low signaling ratio and low Ki67 expression, suggesting that PLK4 serves as a key node between dormancy and proliferation in CRC (Fig. 6C). The percentage of patients 
with high PLK4 expression in the group with $\mathrm{p}-\mathrm{ERK}^{\text {high}} / \mathrm{p}-\mathrm{P} 38^{\text {low }}$ signaling ratio was significantly higher than that in the corresponding group with p-P38 high/ $\mathrm{p}$-ERK ${ }^{\text {low }}$ signaling ratio (Fig. 6D).

Table 2

Univariate and multivariate analysis of clinical parameters on overall survival of CRC patients

\begin{tabular}{|c|c|c|c|c|c|c|}
\hline \multirow[t]{2}{*}{ Clinicopathologic features } & \multicolumn{3}{|c|}{ Univariate analysis } & \multicolumn{3}{|c|}{ Multivariate analysis } \\
\hline & $\begin{array}{l}\text { HR (log } \\
\text { rank) }\end{array}$ & $95 \% \mathrm{Cl}$ & $\begin{array}{l}p \\
\text { value }\end{array}$ & $\begin{array}{l}\text { HR (log } \\
\text { rank) }\end{array}$ & $95 \% \mathrm{Cl}$ & $\begin{array}{l}p \\
\text { value }\end{array}$ \\
\hline Sex (male/female) & 1.094 & $\begin{array}{l}0.557- \\
2.146\end{array}$ & 0.794 & & & \\
\hline Age $(>55 / \leq 55)$ & 1.301 & $\begin{array}{l}0.622- \\
2.723\end{array}$ & 0.482 & & & \\
\hline $\operatorname{CEA}(>5 / \leq 5)$ & 3.765 & $\begin{array}{l}1.857- \\
7.631\end{array}$ & $<.001$ & 2.290 & $\begin{array}{l}1.079- \\
4.860\end{array}$ & 0.031 \\
\hline Tumor size $(>5 / \leq 5)$ & 1.206 & $\begin{array}{l}0.615- \\
2.363\end{array}$ & 0.585 & & & \\
\hline Vascular invasion (yes/no) & 3.265 & $\begin{array}{l}1.517- \\
7.027\end{array}$ & 0.001 & 1.954 & $\begin{array}{l}0.879- \\
4.342\end{array}$ & 0.100 \\
\hline $\begin{array}{l}\text { Lymph nodes metastasis } \\
\text { (yes/no) }\end{array}$ & 2.595 & $\begin{array}{l}1.318- \\
5.108\end{array}$ & 0.004 & 1.520 & $\begin{array}{l}0.680- \\
3.396\end{array}$ & 0.308 \\
\hline Tumor capsule (yes/no) & 2.293 & $\begin{array}{l}0.998- \\
5.268\end{array}$ & 0.044 & 0.951 & $\begin{array}{l}0.355- \\
2.549\end{array}$ & 0.921 \\
\hline Tumor location (left/right) & 2.259 & $\begin{array}{l}1.131- \\
4.513\end{array}$ & 0.018 & 1.945 & $\begin{array}{l}0.953- \\
3.967\end{array}$ & 0.067 \\
\hline TNM stage (III-IV/I-II) & 1.967 & $\begin{array}{l}0.984- \\
3.931\end{array}$ & 0.051 & & & \\
\hline PLK4 expression (high/low) & 4.124 & $\begin{array}{l}1.970- \\
8.635\end{array}$ & $<0.001$ & 3.148 & $\begin{array}{l}1.417- \\
6.997\end{array}$ & 0.005 \\
\hline Ki67 expression (high/low) & 2.528 & $\begin{array}{l}1.232- \\
5.188\end{array}$ & 0.009 & 1.352 & $\begin{array}{l}0.624- \\
2.932\end{array}$ & 0.445 \\
\hline
\end{tabular}

Considering the critical role of PLK4 in CRC dormancy and progression, we further analyzed the correlations between PLK4 and various clinicopathological factors in our CRC cohort. Notably, we found that the expression of PLK4 was markedly correlated with CEA $(p=0.016)$, tumor size $(p=0.041)$, lymph node metastasis status $(p=0.010)$, tumor capsule status ( $p<$ $0.001)$, TMN stage $(p=0.002)$ and Ki67 expression level $(p=0.002)$ (Table 3$)$. Remarkably, the percentage of patients with high PLK4 expression among the high-risk subgroups of patients with an elevated CEA ( $\geq 5 \mathrm{ng} / \mathrm{ml} \mathrm{ng} / \mathrm{ml})$, larger tumor size (>5 cm), positive lymph node metastasis status, ruptured-capsule tumors, advanced TMN stage (stage III \& IV) or high Ki67 expression was significantly higher than that in the corresponding low-risk subgroups (Fig. 6E-J). Consistently, in the highrisk subgroups mentioned above, the CRC patients with low levels of PLK4 expression exhibited a better prognosis than those with high PLK4 expression levels (Fig. 6K-P). Thus, these findings indicate that low expression of PLK4 is a critical protective factor and that PLK4 could serve as a promising biomarker for evaluating tumor dormancy and predicting the prognosis of CRC. 
Table 3

The correlations between PLK4 and various clinicopathological factors in CRC patients

\begin{tabular}{|c|c|c|c|c|c|c|c|c|c|c|c|}
\hline \multirow[t]{2}{*}{ Characteristics } & & \multirow{2}{*}{$\begin{array}{l}\text { Total } \\
122\end{array}$} & \multicolumn{2}{|c|}{$\begin{array}{l}\text { PLK4 } \\
\text { expression }\end{array}$} & \multirow[t]{2}{*}{$\begin{array}{l}p \\
\text { value }\end{array}$} & \multirow[t]{2}{*}{ Characteristics } & & \multirow{2}{*}{$\begin{array}{l}\text { Total } \\
122\end{array}$} & \multicolumn{2}{|c|}{$\begin{array}{l}\text { PLK4 } \\
\text { expression }\end{array}$} & \multirow[t]{2}{*}{$\begin{array}{l}p \\
\text { value }\end{array}$} \\
\hline & & & Low & High & & & & & Low & High & \\
\hline \multirow[t]{3}{*}{ Sex } & & & & & 0.496 & LN metastasis & & & & & 0.010 * \\
\hline & Male & 56 & 34 & 22 & & & yes & 43 & 18 & 25 & \\
\hline & Female & 66 & 36 & 30 & & & no & 79 & 52 & 27 & \\
\hline \multirow[t]{3}{*}{ Age } & & & & & 0.377 & Tumor capsule & & & & & $<.001 *$ \\
\hline & $>55$ & 79 & 43 & 36 & & & no & 78 & 35 & 43 & \\
\hline & $\leq 55$ & 43 & 27 & 16 & & & yes & 44 & 35 & 9 & \\
\hline \multirow[t]{3}{*}{ CEA } & & & & & $0.016^{*}$ & $\begin{array}{l}\text { Tumor } \\
\text { location }\end{array}$ & & & & & 0.839 \\
\hline & $>5$ & 46 & 20 & 26 & & & left & 55 & 31 & 24 & \\
\hline & $\leq 5$ & 76 & 50 & 26 & & & right & 67 & 39 & 28 & \\
\hline \multirow[t]{3}{*}{ Tumor size } & & & & & $0.041 *$ & TNM stage & & & & & $0.002 *$ \\
\hline & $>5$ & 55 & 26 & 29 & & & III-IV & 58 & 25 & 33 & \\
\hline & $\leq 5$ & 67 & 44 & 23 & & & $\mathrm{I}-\mathrm{II}$ & 64 & 45 & 19 & \\
\hline \multirow[t]{3}{*}{ VI } & & & & & 0.241 & $\begin{array}{l}\text { Ki67 } \\
\text { expression }\end{array}$ & & & & & $<.001 *$ \\
\hline & yes & 16 & 7 & 9 & & & high & 59 & 24 & 35 & \\
\hline & no & 106 & 63 & 43 & & & low & 63 & 46 & 17 & \\
\hline \multicolumn{12}{|c|}{ VI: Vascular invasion } \\
\hline LN: Lymph nod & & & & & & & & & & & \\
\hline
\end{tabular}

\section{Discussion}

The functions of PLK family members, including cell cycle regulation and DNA damage response contributions, as well as participation in the carcinogenesis and metastasis of various cancers, are widely understood[28, 29]. Abnormal expression of PLK4, a member of the PLK family, contributes to cell cycle arrest and proliferation impairment, which are the two main characteristics of dormant cells according to previous studies[17, 29, 30]. However, the intrinsic relationship and regulatory mechanisms linking PLK4 and tumor cell dormancy are still largely unknown.

In this study, we demonstrated that the expression of PLK4 was dramatically increased in CRC and positively correlated with aggressive tumor behavior and clinicopathological risk factors by using analysis of a large-scale CRC clinical sample cohort and public online datasets. Moreover, it should be emphasized that our findings are consistent with those of prior studies[22], implying that PLK4 expression is upregulated in cancers and associated with biological aggressiveness and poor patient outcomes[18]. Furthermore, subsequent studies have indicated that PLK4 deficiency in CRC cells induces the MET process with impairment of migration and invasion in vitro and in vivo, which is in agreement with previous studies of neuroblastoma and breast cancer[20, 21]. Interestingly, we further revealed a link between PLK4 and tumor cell dormancy, which had not been reported before. In our study, we found that loss of PLK4 expression induced CRC cells to not only 
transform into an epithelial phenotype but also exhibit characteristics of dormant cells, such as G0/G1 cell cycle arrest and reduced proliferation and clone formation rates. In addition, further evidence provided by immunohistochemical staining of CRC clinical samples showed that PLK4 deficiency was associated with low Ki67 expression and p-P38 ${ }^{\text {high }} / \mathrm{p}^{-E} \mathrm{EK}^{\text {low }}$ signaling ratio. However, when TGF- $\beta$ was employed to reverse the MET process caused by the downregulation of PLK4 expression, the dormant phenotype of the PLK4-depleted cells was also lost as the cells were reactivated. From these observations, we propose that downregulation of PLK4 expression contributes to restoring phenotypically aggressive tumor cells to a quiescent state and that this transformation is likely regulated by MET progression.

In fact, the relationships between EMT or MET and tumor cell dormancy have attracted great attention for a long time. Consistent with our findings, Kathryn L. Harper et al. reported that a subpopulation of early-disseminated Her $2^{+}$cancer cells activated a Wnt-dependent EMT-like dissemination program, but without complete loss of the epithelial phenotype, to form a metastatic lesion after a dormant phase[31]. Furthermore, a study by Simon A Joosse et al. suggested that once disseminating tumor cells arrive at a distant site, they may extravasate, undergo MET, and remain dormant[32]. In contrast, there is also evidence that EMT promotes tumor cell dormancy. For example, Federica Francescangeli et al. identified a preexisting population of ZEB2 ${ }^{+}$quiescent cells with stemness and mesenchymal phenotypic features that dictate chemoresistance in CRC[33]. All these findings greatly supplement our knowledge of tumor cell dormancy. Considering the complexity of metastasis and relapse as well as the heterogeneity among cancers, it is also possible that EMT and MET differentially regulate tumor cell dormancy in different tumor types or metastatic cascades. However, in our study, we confirmed that silencing PLK4 in CRC cells induced MET, which resulted in tumor cell dormancy. Furthermore, it is noteworthy that the occurrence of this event was found to be beneficial to the prognosis of patients with CRC.

Whether tumor cell dormancy is a challenge or an opportunity in clinical cancer treatment remains controversial. Currently, the pharmacological strategies for targeting dormant tumor cells include three approaches[34]. First is the eradication of dormant cancer cells. Kari J. Kurppa et al. confirmed that eradicating tumor dormancy following drug treatment is an effective therapeutic strategy to reduce relapse and improve treatment outcomes in EGFR-mutant non-small cell lung cancer[33]. Second is the reactivation of dormant cells reactivated to increase their susceptibility to antiproliferative drugs. For example, forcing quiescent residual leukemic cells to reenter the cell cycle by employing granulocyte colony-stimulating factor (G-CSF) is considered a useful therapeutic strategy for acute myeloid leukemia (AML)[35]. Third is the maintenance of tumor cells in the dormant state. In ER ${ }^{+}$breast cancers, adjuvant anti-estrogen therapy has been widely used to inhibit dormant cell outgrowth, which effectively prolongs patient survival[36]. In accordance with the last strategy, potential therapies that reduce PLK4 expression by using specific inhibitors to keep tumor cells in the harmless dormant state may provide opportunities to prolong the therapeutic window and achieve better survival in CRC patients.

\section{Conclusion}

In summary, PLK4 is an independent prognostic factor in CRC. Downregulation of PLK4 expression in CRC cells promotes G0/G1 cell cycle arrest and impairs proliferation, migration and invasion through the MET process in vivo, which suggests the development of a dormant phenotype. Further evidence reveals that these tumor cells induced to become dormant by PLK4 depletion partially lose the capacity to form in situ lesions and distal metastases in CRC xenograft model mice. Furthermore, the expression level of PLK4 can be applied to identify the dormant state of CRC according to the results of immunohistochemical staining of clinical samples, which is significant for estimating the prognosis of CRC patients. This study highlights the role of PLK4 as a biomarker for CRC prognosis and suggests a promising future for PLK4 inhibitors as potential dormancy-associated- therapeutic agents for CRC therapy.

\section{Declarations}

\section{Consent for publication}

Page $12 / 20$ 


\section{Availability of data and materials}

The datasets used during the current study are available from the corresponding author on reasonable request.

\section{Competing interests}

The authors declare that they have no competing interests.

\section{Funding}

This work was supported by the grants from National Natural Science Foundation of China (81903055 to X.T., 81902401 to L.C). National Key R\&D Program of China (2018YFC1313000 to Q.Z.). Key Project of Tianjin Natural Science Foundation (18JCZDJC35200 to H.G.). Science \& Technology Development Fund of Tianjin Education Commission for Higher Education (2017KJ202 to L.C.). Scientific Research Foundation of Tianjin Medical University Cancer Institute and Hospital (1706 to X.T., B1703 to L.C., B1701 to W.G.). L.C. is also supported by China Scholarship Council (CSC.201906940003) and Heng Rui Research Grant for Hepatobiliary and Pancreatic Cancer (CXPJJH11800001-2018316).

\section{Authors' Contributions}

HG, QZ LC and TX designed the research studies; TX DL, ZH, ZC and QD conducted experiments; DL analyzed the clinical data; LQ and WG performed pathological diagnosis and evaluation; HL, JW and PL collected clinical samples; YL and LC helped with the retouching of the article language; LC wrote the manuscript; HG revised the manuscript.

\section{Acknowledgements}

We thank all the researchers from the Department of Tumor Cell Biology, Tianjin Medical University Cancer Institute and Hospital. L.C. would also like to thank the financial support from China Scholarship Council (CSC).

\section{References}

1. Ladabaum U, Dominitz JA, Kahi C, Schoen RE. Strategies for Colorectal Cancer Screening. Gastroenterology. 2020;158(2):418-32.

2. Siegel RL, Miller KD, Jemal A. Cancer statistics. 2019. CA Cancer J Clin. 2019. 69(1): 7-34.

3. van der Stok EP, Spaander M, Grünhagen DJ, Verhoef C, Kuipers EJ. Surveillance after curative treatment for colorectal cancer. Nat Rev Clin Oncol. 2017;14(5):297-315.

4. Dienstmann R, Villacampa G, Sveen A, et al. Relative contribution of clinicopathological variables, genomic markers, transcriptomic subtyping and microenvironment features for outcome prediction in stage II/III colorectal cancer. Ann Oncol. 2019;30(10):1622-9.

5. Dekker E, Rex DK. Advances in CRC Prevention: Screening and Surveillance. Gastroenterology. 2018;154(7):1970-84.

6. Chen L, Liu D, Yi X, et al. The novel miR-1269b-regulated protein SVEP1 induces hepatocellular carcinoma proliferation and metastasis likely through the PI3K/Akt pathway. Cell Death Dis. 2020;11(5):320.

7. Sosa MS, Bragado P, Aguirre-Ghiso JA. Mechanisms of disseminated cancer cell dormancy: an awakening field. Nat Rev Cancer. 2014;14(9):611-22. 
8. Elias D, Viganò L, Orsi F, et al. New Perspectives in the Treatment of Colorectal Metastases. Liver Cancer. 2016;6(1):908.

9. Demicheli R. Tumour dormancy: findings and hypotheses from clinical research on breast cancer. Semin Cancer Biol. 2001;11(4):297-306.

10. Chambers AF, Groom AC, MacDonald IC. Dissemination and growth of cancer cells in metastatic sites. Nat Rev Cancer. 2002;2(8):563-72.

11. Aguirre-Ghiso JA. Models, mechanisms and clinical evidence for cancer dormancy. Nat Rev Cancer. 2007;7(11):834-46.

12. Giancotti FG. Mechanisms governing metastatic dormancy and reactivation. Cell. 2013;155(4):750-64.

13. Ruppender NS, Morrissey C, Lange PH, Vessella RL. Dormancy in solid tumors: implications for prostate cancer. Cancer Metastasis Rev. 2013;32(3-4):501-9.

14. Adam AP, George A, Schewe D, et al. Computational identification of a p38SAPK-regulated transcription factor network required for tumor cell quiescence. Cancer Res. 2009;69(14):5664-72.

15. Aguirre-Ghiso JA, Ossowski L, Rosenbaum SK. Green fluorescent protein tagging of extracellular signal-regulated kinase and p38 pathways reveals novel dynamics of pathway activation during primary and metastatic growth. Cancer Res. 2004;64(20):7336-45.

16. Rodrigues-Martins A, Riparbelli M, Callaini G, Glover DM, Bettencourt-Dias M. Revisiting the role of the mother centriole in centriole biogenesis. Science. 2007;316(5827):1046-50.

17. Habedanck R, Stierhof YD, Wilkinson CJ, Nigg EA. The Polo kinase Plk4 functions in centriole duplication. Nat Cell Biol. 2005;7(11):1140-6.

18. Yamamoto S, Kitagawa D. Self-organization of Plk4 regulates symmetry breaking in centriole duplication. Nat Commun. 2019;10(1):1810.

19. Rosario CO, Ko MA, Haffani YZ, et al. Plk4 is required for cytokinesis and maintenance of chromosomal stability. Proc Natl Acad Sci U S A. 2010;107(15):6888-93.

20. Tian X, Zhou D, Chen L, et al. Polo-like kinase 4 mediates epithelial-mesenchymal transition in neuroblastoma via PI3K/Akt signaling pathway. Cell Death Dis. 2018;9(2):54.

21. Kazazian K, Go C, Wu H, et al. Plk4 Promotes Cancer Invasion and Metastasis through Arp2/3 Complex Regulation of the Actin Cytoskeleton. Cancer Res. 2017;77(2):434-47.

22. Liao Z, Zhang H, Fan P, et al. High PLK4 expression promotes tumor progression and induces epithelial-mesenchymal transition by regulating the Wnt/ $\beta$-catenin signaling pathway in colorectal cancer. Int J Oncol. 2019;54(2):479-90.

23. Liu D, Lin L, Wang Y, Chen L, He Y, Luo Y, Qi L, Guo Y, Chen L, Han Z, et al. PNO1, which is negatively regulated by miR340-5p, promotes lung adenocarcinoma progression through Notch signaling pathway. Oncogenesis. 2020;9:58.

24. Aguirre-Ghiso JA, Estrada Y, Liu D, Ossowski L. ERK(MAPK) activity as a determinant of tumor growth and dormancy; regulation by p38(SAPK). Cancer Res. 2003;63(7):1684-95.

25. Sosa MS, Avivar-Valderas A, Bragado P, Wen HC, Aguirre-Ghiso JA. ERK1/2 and p38a/ $\beta$ signaling in tumor cell quiescence: opportunities to control dormant residual disease. Clin Cancer Res. 2011;17(18):5850-7.

26. Jensen KB, Watt FM. Single-cell expression profiling of human epidermal stem and transit-amplifying cells: Lrig1 is a regulator of stem cell quiescence. Proc Natl Acad Sci U S A. 2006;103(32):11958-63.

27. Chen L, Fu H, Luo Y, et al. cPLA2a mediates TGF- $\beta$-induced epithelial-mesenchymal transition in breast cancer through PI3k/Akt signaling. Cell Death Dis. 2017;8(4):e2728.

28. Takai N, Hamanaka R, Yoshimatsu J, Miyakawa I. Polo-like kinases (PIks) and cancer. Oncogene. 2005;24(2):287-91.

29. Nigg EA. Centrosome duplication: of rules and licenses. Trends Cell Biol. 2007;17(5):215-21.

30. Gemble S, Basto R. Fast and furious... or not, Plk4 dictates the pace. J Cell Biol. 2018. 217(4): 1169-1171. 
31. Harper KL, Sosa MS, Entenberg D, et al. Mechanism of early dissemination and metastasis in Her2 + mammary cancer. Nature. 2016;540(7634):588-92.

32. Joosse SA, Gorges TM, Pantel K. Biology, detection, and clinical implications of circulating tumor cells. EMBO Mol Med. 2015;7(1):1-11.

33. Francescangeli F, Contavalli P, De Angelis ML, et al. A pre-existing population of ZEB2 + quiescent cells with stemness and mesenchymal features dictate chemoresistance in colorectal cancer. J Exp Clin Cancer Res. 2020;39(1):2.

34. Recasens A, Munoz L. Targeting Cancer Cell Dormancy. Trends Pharmacol Sci. 2019;40(2):128-41.

35. Di Tullio A, Rouault-Pierre K, Abarrategi A, et al. The combination of CHK1 inhibitor with G-CSF overrides cytarabine resistance in human acute myeloid leukemia. Nat Commun. 2017;8(1):1679.

36. Abderrahman B, Jordan VC. Rethinking Extended Adjuvant Antiestrogen Therapy to Increase Survivorship in Breast Cancer. JAMA Oncol. 2018;4(1):15-6.

\section{Figures}
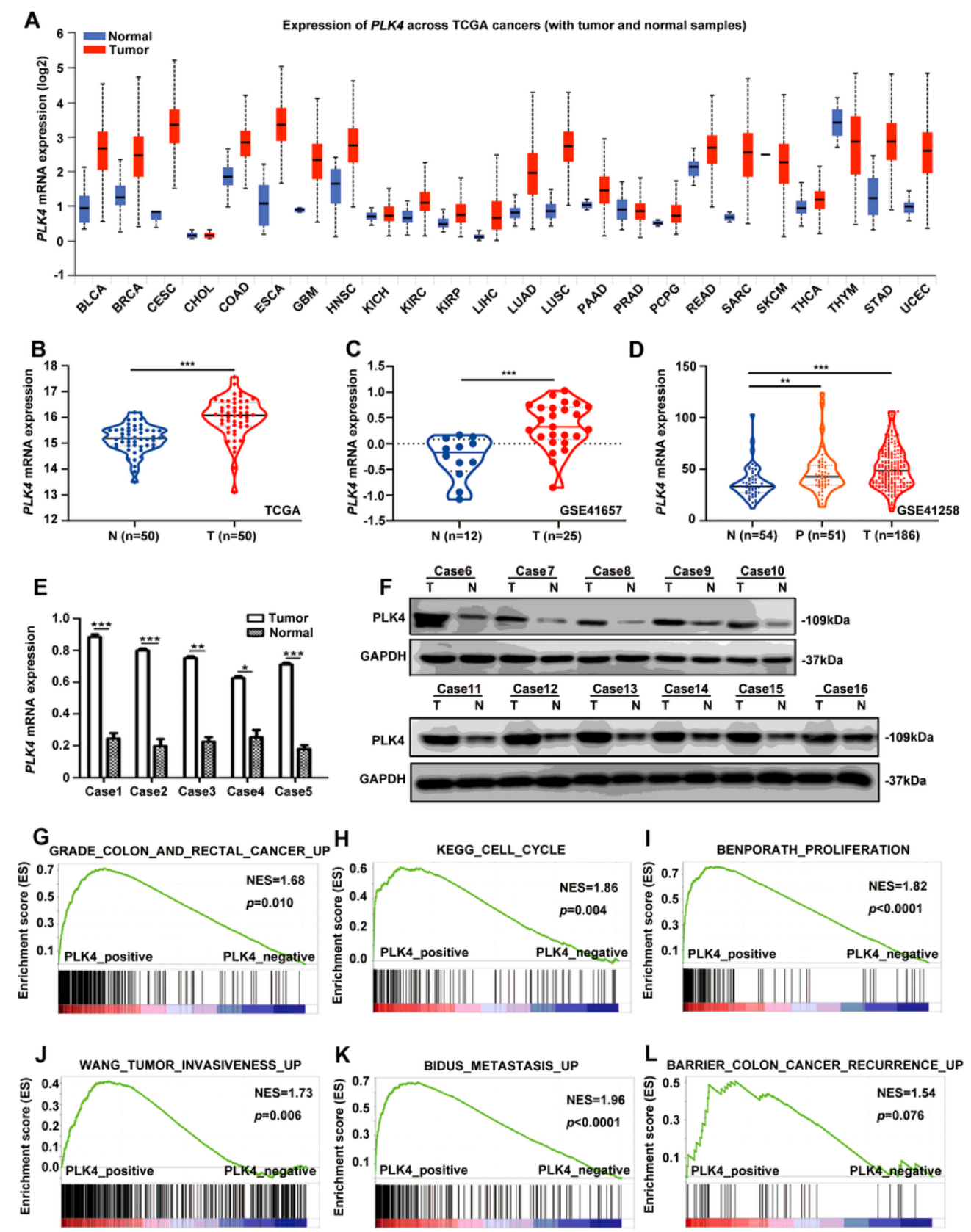
Figure 1

PLK4 is overexpressed in CRC tissues and associated with aggressive tumor behavior. A. Enhanced PLK4 expression was observed in tumor tissues compared with adjacent normal tissues in most cancer types, including CRC. B-D. The expression of PLK4 was examined in CRC tissue samples and adjacent nonmalignant tissue samples from the TCGA and GEO databases (N: normal, T: tumor, and P: polyp). E: qRT-PCR showed differences in PLK4 mRNA levels in five pairs of CRC tissue and adjacent nonmalignant tissue. F: WB showed differences in PLK4 protein levels in eleven pairs of CRC tissue and adjacent nonmalignant tissue. G-L: GSEA was used to analysis the associations of high PLK4 expression in CRC with a series of malignant behaviors linked to an unfavorable prognosis in CRC. ${ }^{*} P<0.05$, ${ }^{*} P<0.01, * \star * P<0.001$.

A

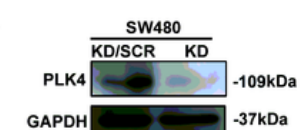

GAPDH $\longrightarrow-37 \mathrm{kDa}$
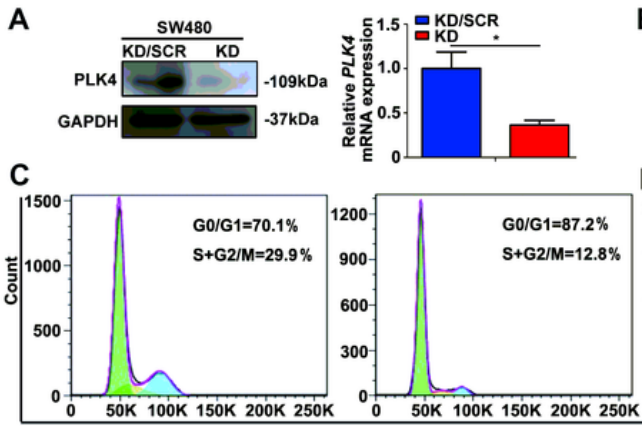

B
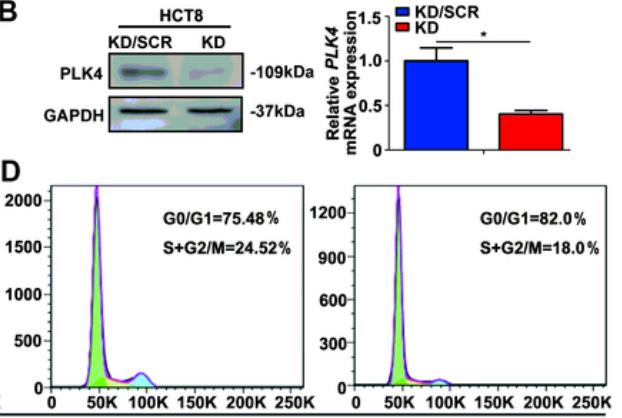

E
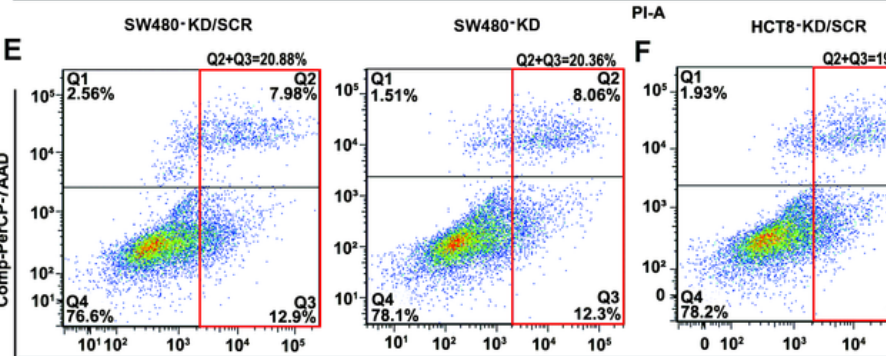

HCT8·KD
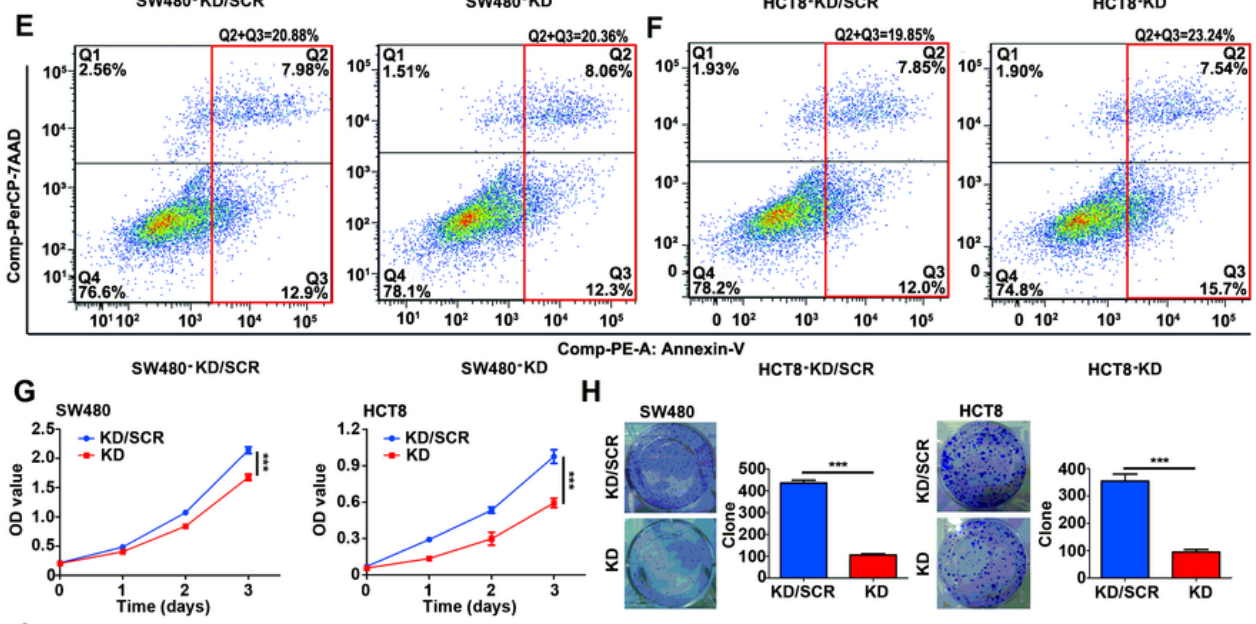

H
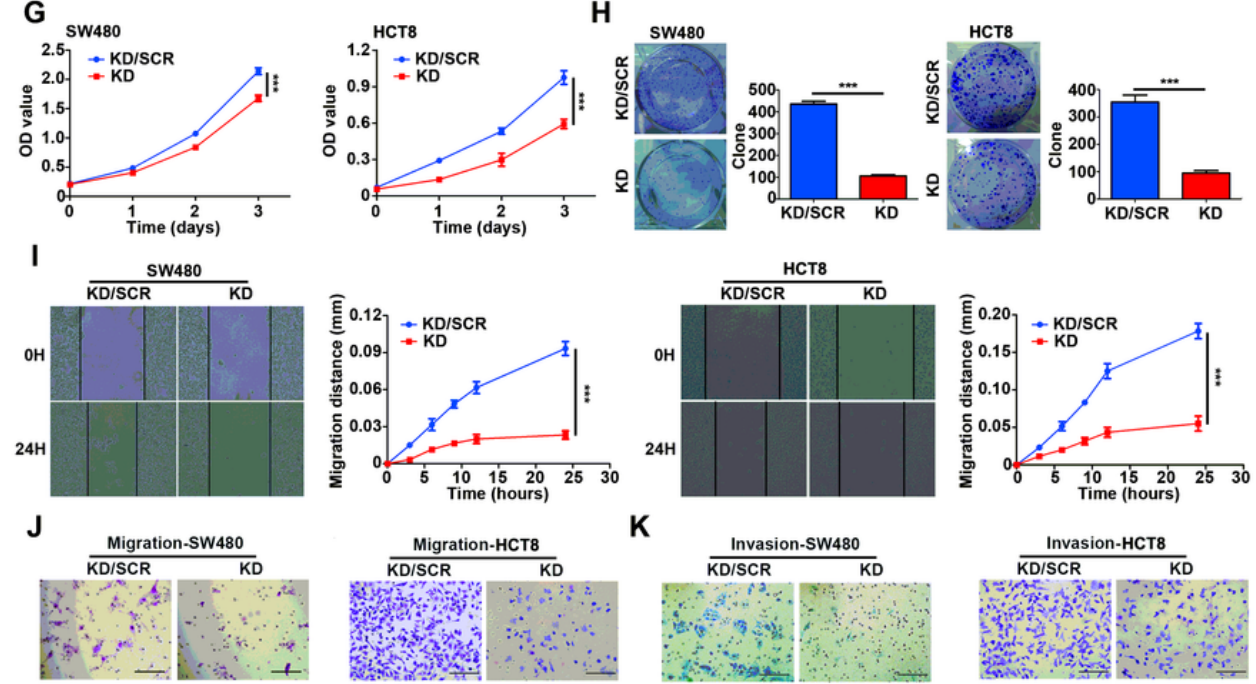

K
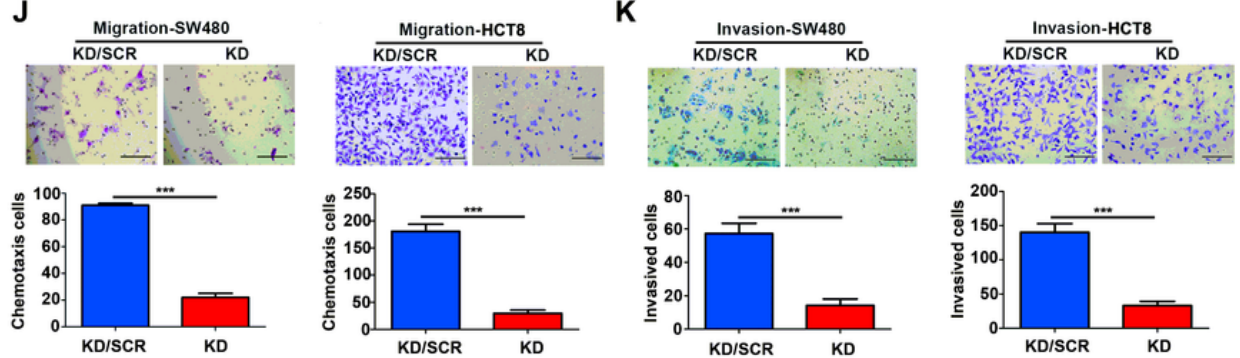

Figure 2

PLK4 is essential in the cell cycle, proliferation, migration and invasion of CRC cells. A-B. The construction of PLK4-control (KD/SCR) and PLK4-knockdown (KD) SW480 and HCT8 CRC cell lines. C-D. Cell cycle assay revealing the difference in the G0/G1 cell cycle proportion between the KD/SCR and KD groups of CRC cell lines. E-F. Cell apoptosis assay revealing the 
proportions of apoptotic SW480 and HCT8 cells in the KD/SCR and KD groups. G-H. CCK-8 and clone formation assays revealing the proliferative abilities of SW480 and HCT8 cells in the KD/SCR and KD groups. I-K. Wound-healing, chemotaxis and transwell assays comparing cell migration and invasion differences between the KD/SCR and KD groups of SW480 and HCT8 cells (scale bar, $1.0 \mathrm{~mm}$ ). ${ }^{*} \mathrm{P}<0.05,{ }^{*} * \mathrm{P}<0.001$.

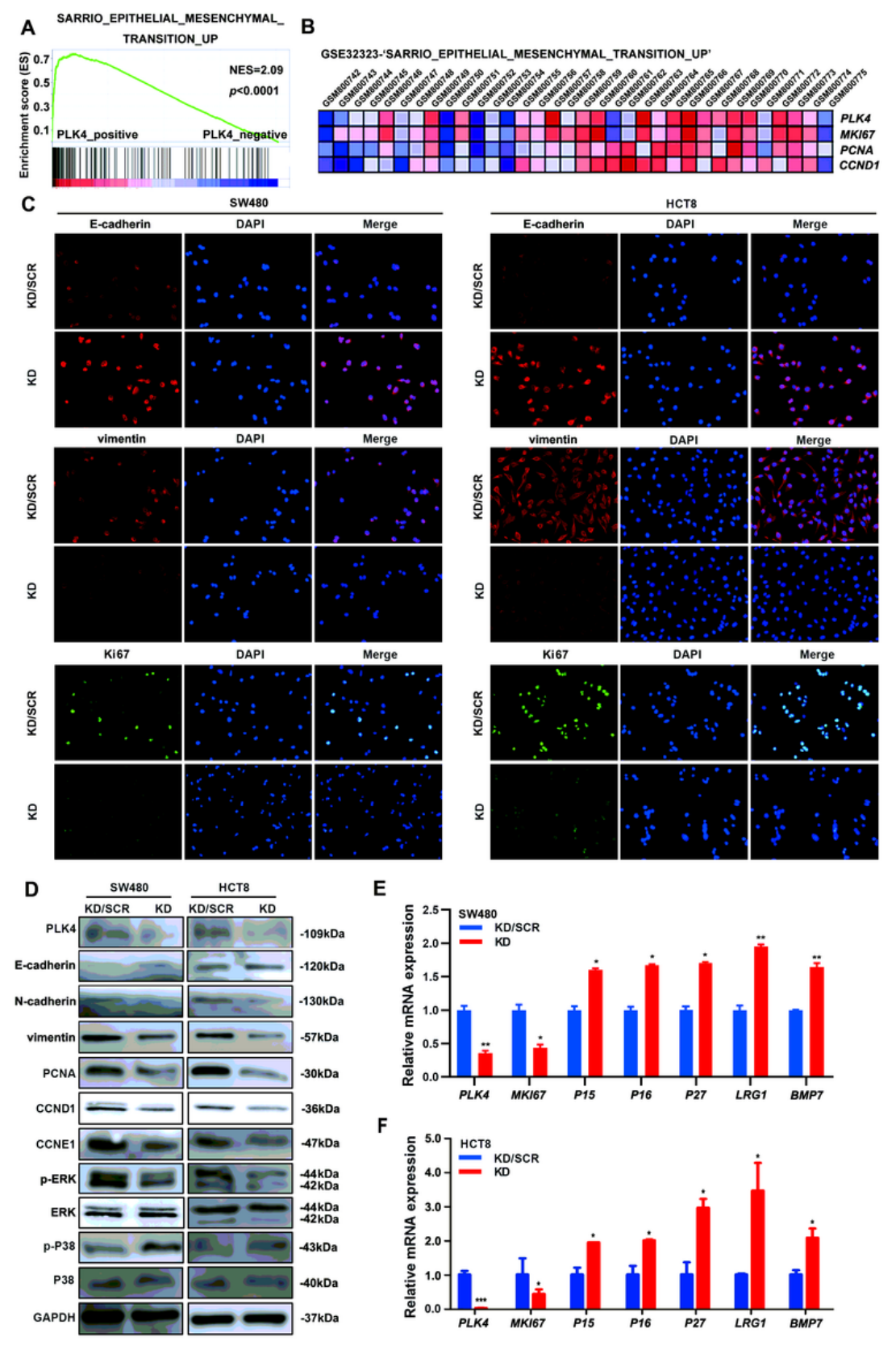

Figure 3

Knockdown of PLK4 expression promotes epithelial and dormant phenotypic transformation in CRC cells. A-B. GSEA showing the relationships between PLK4 expression and EMT, proliferation and cell cycle genes. C. Immunofluorescence assay revealing that downregulation of PLK4 expression induced epithelial phenotypic transformation in CRC cells. D. The protein levels of PLK4 and EMT, cell proliferation and cell cycle markers as assessed by WB. E-F. qRT-PCR assay evaluating Signaling molecules favoring the acquisition of a dormant phenotype in the KD/SCR and KD groups of SW480 and HCT8 cells. ${ }^{*} \mathrm{P}<0.05,{ }^{* *} \mathrm{P}<0.01$. 


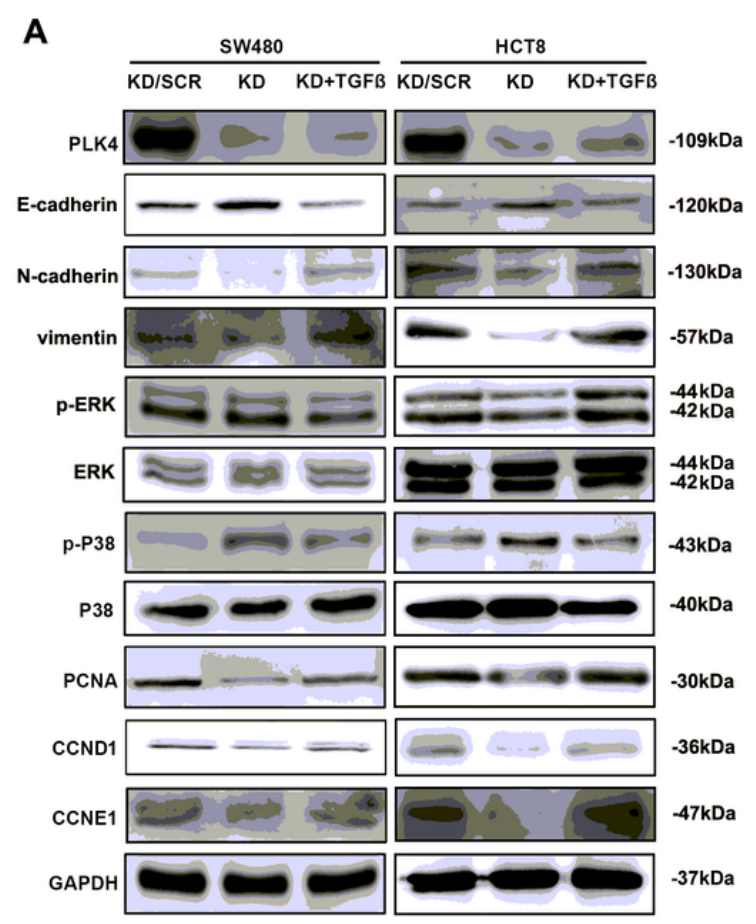

B $S W 480 \cdot K D / S C R \quad S W 480 \cdot K D \quad S W 480 \cdot K D+T G F B$

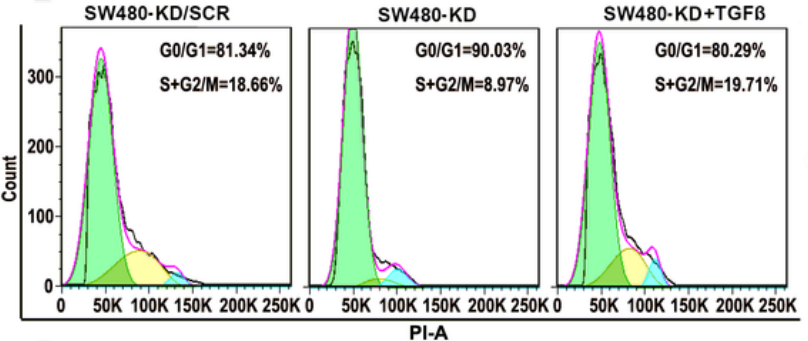

C

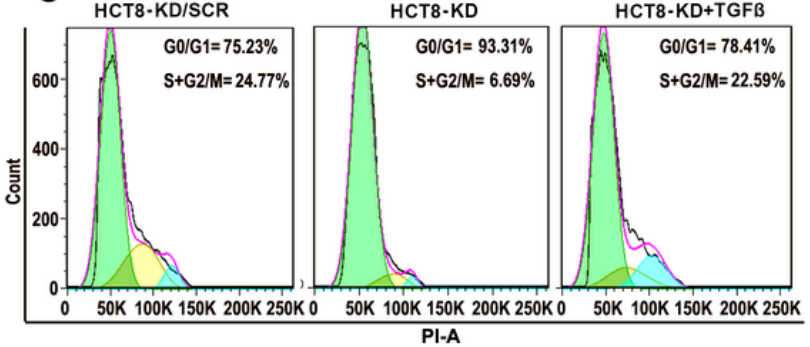

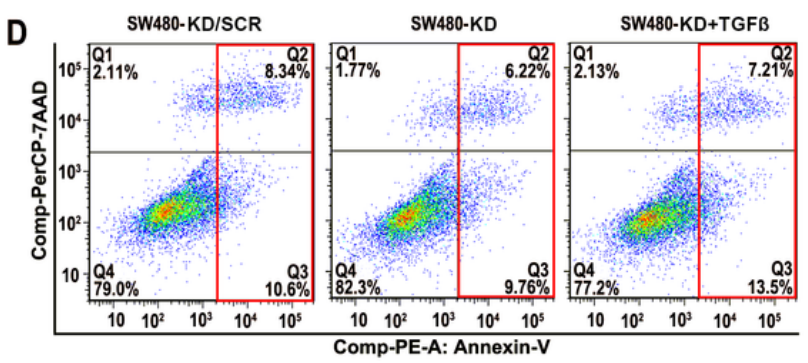
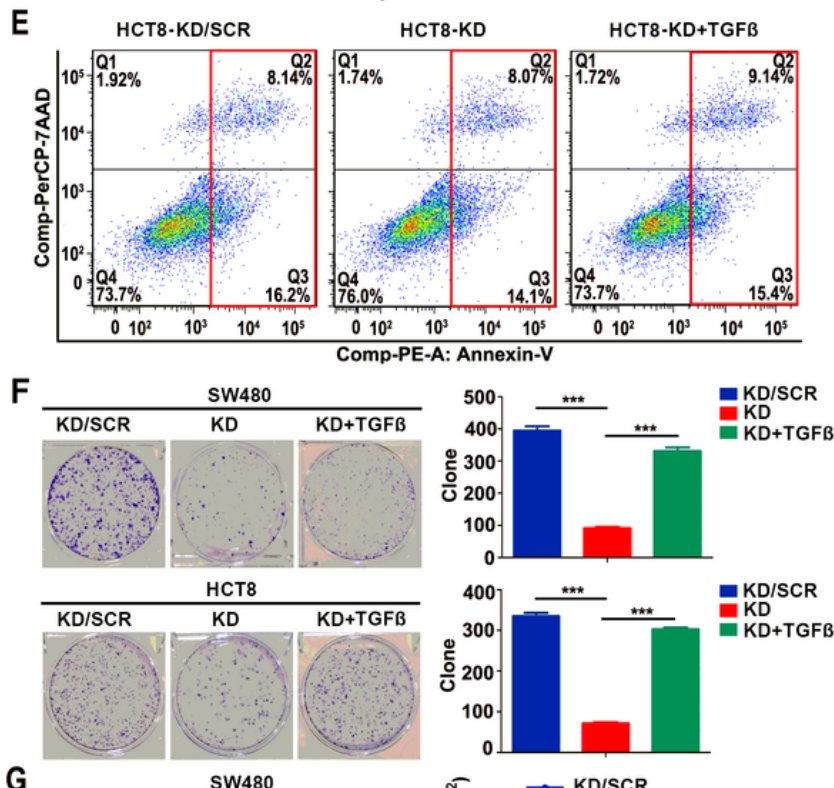

G
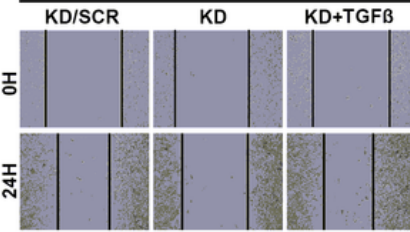

E $147 \mathrm{KD} / \mathrm{SCR}$
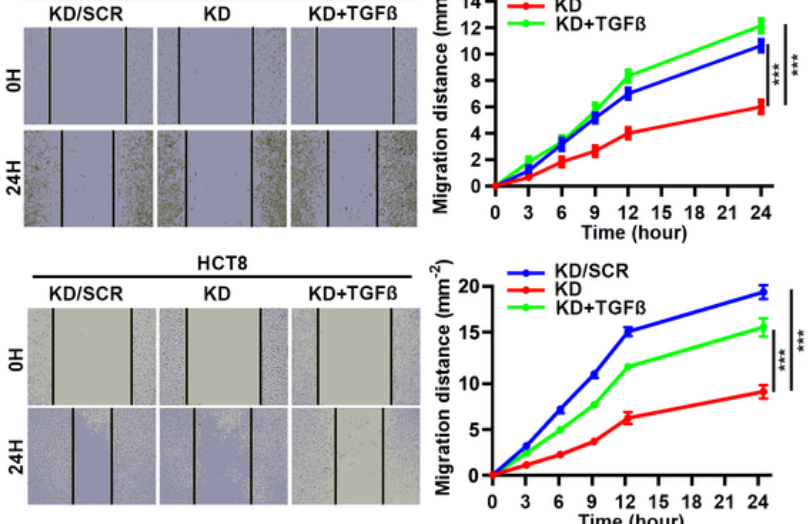

Figure 4

MET reversal reactivates dormant PLK4 depleted CRC cells. A. TGF- $\otimes$ reverses MET and reactivates CRC cell dormancy in CRC cells, as confirmed by WB. B-C. A cell cycle assay revealed cell cycle rescue occurred in PLK4-depleted CRC cells

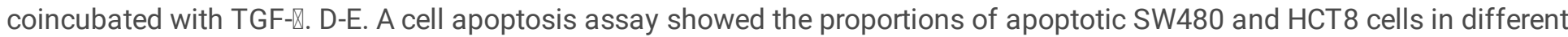

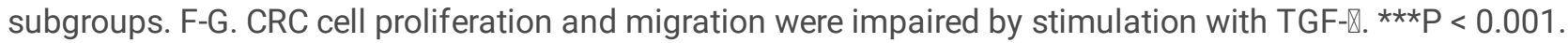




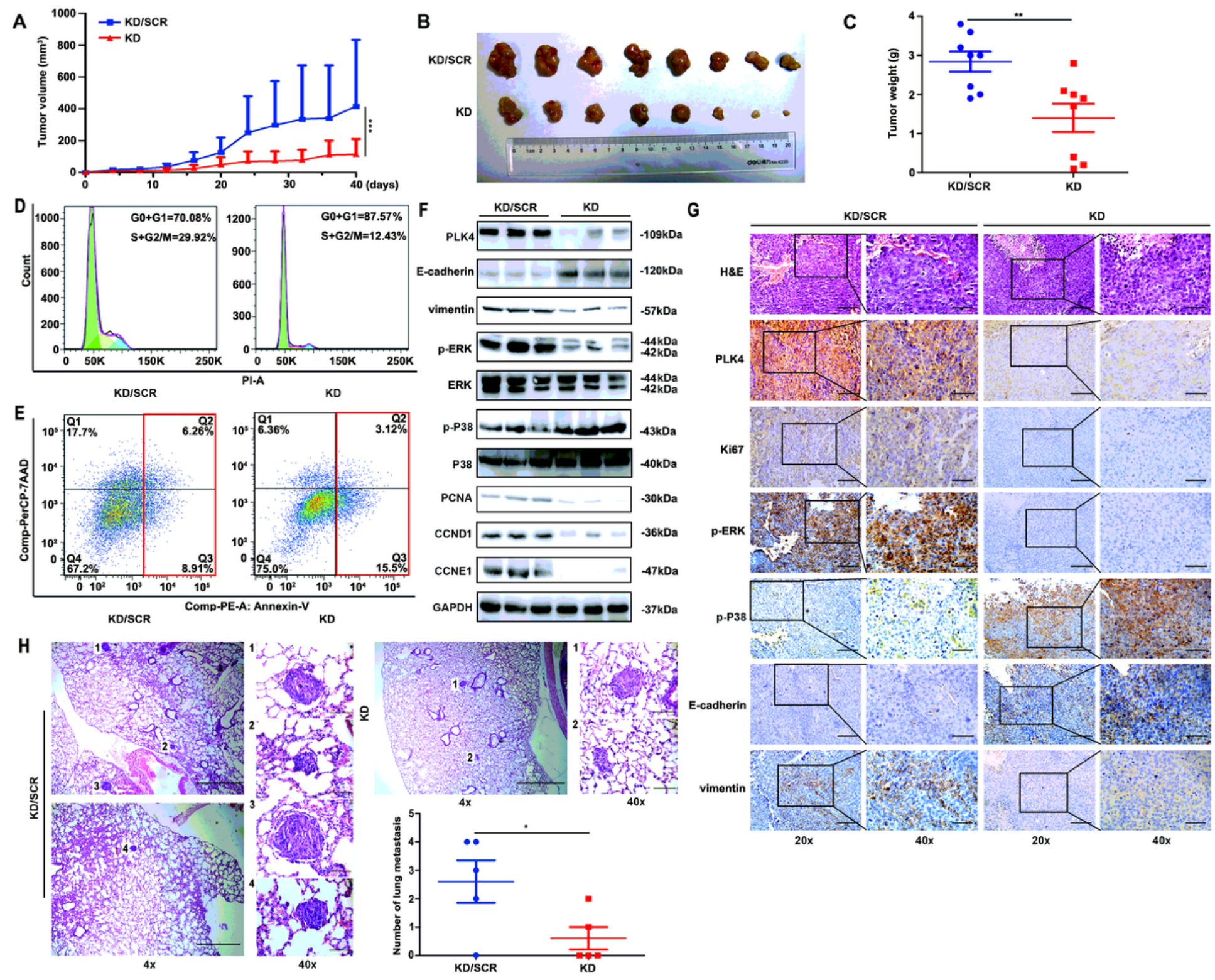

Figure 5

Silencing PLK4 in CRC cells facilitates dormancy and impairs metastasis in vivo. A. The primary tumor growth rates of the two groups. B, C. Tumor volumes and weights in the KD/SCR and KD groups. D-E. Cell cycle and apoptosis differences between the two groups. F. The protein levels of PLK4 and EMT, cell proliferation and cell cycle markers in the xenograft model, as assessed by WB. G. Verification of PLK4 and cell proliferation, cell cycle and EMT markers in the xenograft model, as assessed by IHC; $20 \times$ and $40 \times$. H. H\&E staining of lung metastasis in tail vein-injected mice ( $4 \times$ and $40 \times$ ), and the numbers of lung metastasis nodules in the KD/SCR and KD groups. ${ }^{*} P<0.05,{ }^{*} P<0.01, * * * P<0.001$. 


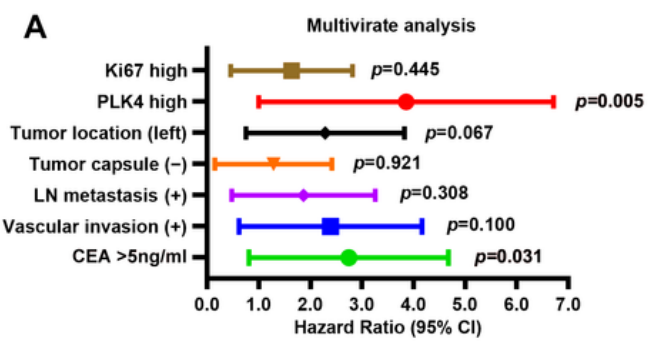

C

D high p-ERK/p-P38 — low p-ERK/p-P38
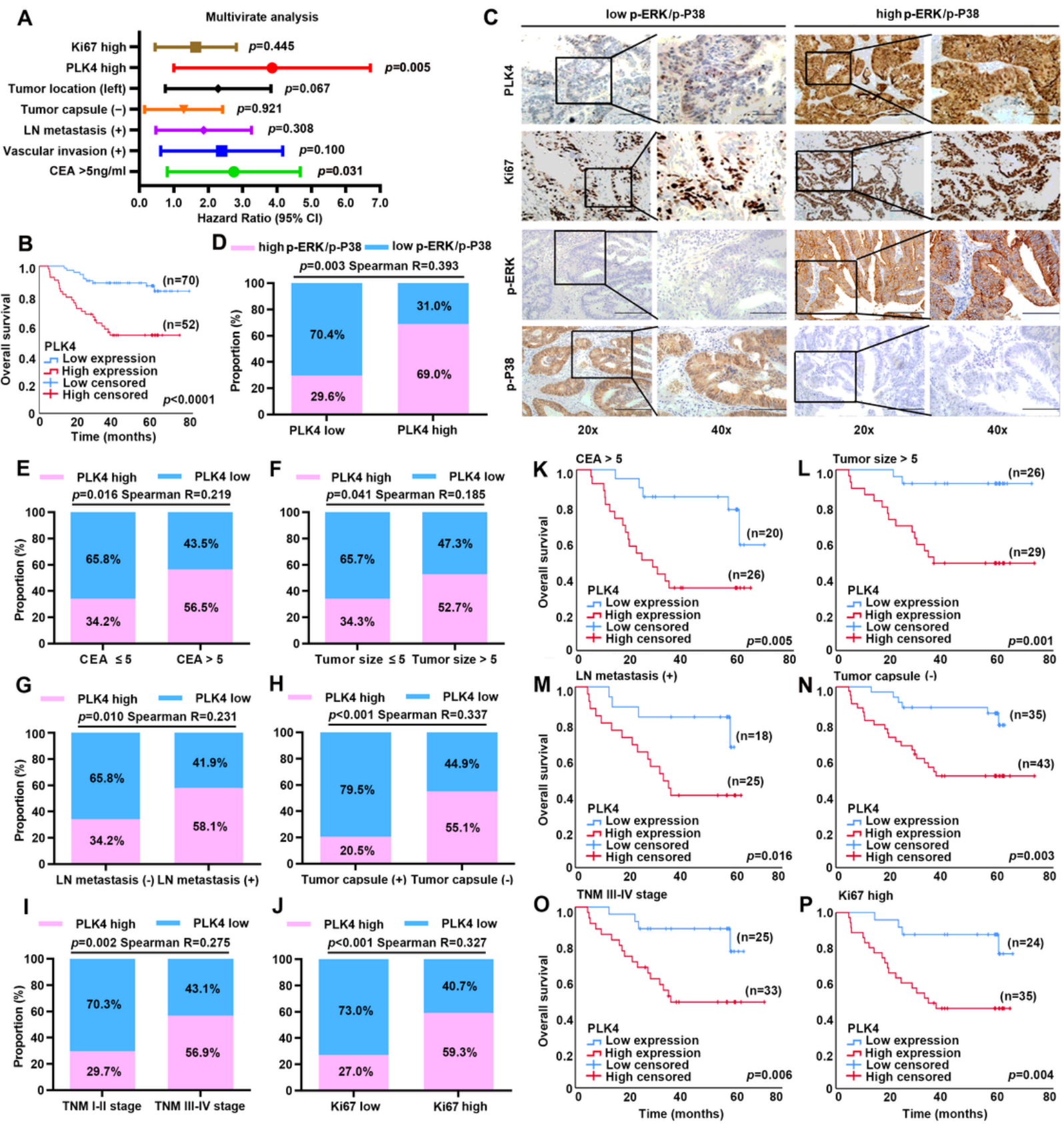

Figure 6

PLK4 inactivation identifies tumor dormancy and predicts a good prognosis in CRC. A. Multivariate analysis identifying PLK4 as an independent risk factor for the prognosis of CRC. B. Kaplan-Meier survival curve validating the correlation between the PLK4 expression level and CRC patient OS. C. IHC revealing PLK4 as a key node between dormancy and proliferation in CRC, 20x and 40x. D-J. Correlation analysis between the PLK4 IHC score and clinicopathological factors in the CRC cohort. K-P. Kaplan-Meier survival curve showing the correlation between the PLK4 IHC score and OS of the high-risk subgroup in the CRC cohort. 